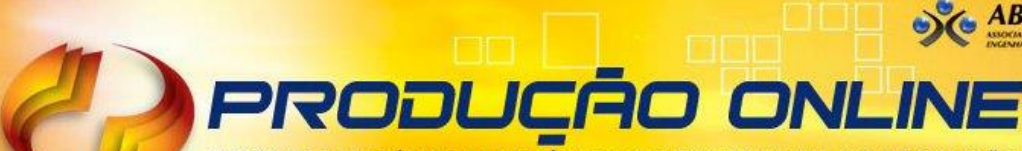 \\ REVISTA CIENTIFICA ELETRÓNICA DE ENGENHARIA DE PRODUÇÃO \\ ISSN 1676-1901
}

\section{OTIMIZAÇÃO DA ROTA DE VEÍCULOS E SEUS IMPACTOS NA LOGÍSTICA DE DISTRIBUIÇÃO: UM ESTUDO DE CASO EM UMA DISTRIBUIDORA DE PRODUTOS DE LIMPEZA}

\section{OPTIMIZATION OF THE ROUTE OF VEHICLES AND THEIR IMPACTS IN DISTRIBUTION LOGISTICS: A CASE STUDY IN A CLEANING PRODUCTS DISTRIBUIDOR}

\author{
Matheus das Neves Almeida* E-mail: matheusalmeida@ufpi.edu.br \\ Belchior Rodrigues dos Santos Neto* E-mail: belchiornrodrigues@gmail.com \\ Agnelo Matos de Moura Filho* E-mail: agnelo50@hotmail.com \\ Arnaldo Alves dos Santos* E-mail: arnaldo.santtos@hotmail.com \\ *Universidade Federal do Piauí (UFPI), Teresina, PI
}

\begin{abstract}
Resumo: Em decorrência de um mercado cada vez mais dinâmico e competitivo, as atividades logísticas ganham destaque como diferencial competitivo em uma organização, exigindo sistemas de distribuição cada vez mais eficientes, posto que os custos de transporte representam a maior parcela dentre os custos logísticos. Nesse contexto, a presente pesquisa visa otimizar a rota de veículos em uma distribuidora de produtos de limpeza localizada. Dessa forma, a metodologia empregada nesse trabalho consiste em um estudo de caso, no qual os dados foram coletados por meio de entrevistas semiestruturadas realizadas com o gestor e demais colaboradores em conjunto ao acesso as informações do sistema de ERP da organização. Após a obtenção dos dados, estes foram tabulados em planilhas eletrônicas do Microsoft Excel para uma posterior análise por meio de um comparativo entre a rota atual e a otimizada pelo software logístico LOGWARE. Os resultados alcançados indicam que as empresas podem obter benefícios e melhorias significativas na logística de distribuição, que para esse caso específico analisado durante 18 dias promoveu uma redução no deslocamento de $183,5 \mathrm{~km}$, no tempo de atendimento de $6,1 \mathrm{~h}$ e ainda, uma economia monetária de aproximadamente $12,34 \%$ em seus custos variáveis. Portanto, conclui-se que é de fundamental importância o uso de métodos computacionais no auxílio da elaboração de rota de distribuição em uma empresa, especialmente considerando a importância dos custos de transportes no custo de logístico total.
\end{abstract}

Palavras-chave: Logística de distribuição. Transporte. Otimização de rotas.

Abstract: As a result of an increasingly dynamic and competitive market, logistics activities are highlighted as a competitive differential in an organization, requiring more and more efficient distribution systems, since transport costs represent the largest share among logistics costs. In this context, the present research aims to optimize the route of vehicles in a distributor of cleaning product. This way, the methodology used in this work consists of a case study, in which data were collected through semi-structured interviews conducted with the manager and other collaborators, along with the access to information from the ERP system of the organization. After obtaining the data, these were tabulated in Microsoft Excel spreadsheets for further analysis through a comparison between the current and optimized route by LOGWARE logistical software. The results obtained indicate that companies can obtain significant benefits and improvements in the distribution logistics, which for this specific case analyzed during 18 days promoted a reduction in the displacement of $1487.3 \mathrm{~km}$ to $1303.8 \mathrm{~km}$, in the service time of 20 min and also, a monetary economy of approximately $12.34 \%$ in their variable costs. Therefore, it is concluded that it is of fundamental importance the use of computational methods to aid in the elaboration of a distribution route in a company, especially considering the importance of transport costs in the total logistics cost. 
Keywords: Distribution Logistics. Transport. Optimization of routes.

\section{INTRODUÇÃO}

Tendo em vista que o mercado se encontra gradativamente mais dinâmico e competitivo, as organizações visam maior agilidade no fluxo das informações e mercadorias, dessa forma, compreende-se a importância da logística de distribuição, em especial, o processo de transporte e entrega dos produtos (NOVAES, 2015).

Dessa forma, entende-se que a eficiência do sistema logístico tornou-se uma condição básica para a competitividade em uma organização, corroborando com isso, DORNIER et al. (2011) afirmam que as mudanças tecnológicas e os mercados emergentes forçaram as empresas a reorganizar, adaptar e otimizar o fluxo de mercadorias. Dessa forma, o desafio compreende não apenas na qualidade dos produtos e na capacidade de inovar, mas também na atenção quanto a disponibilidade e no atendimento aos prazos de entrega dos produtos, fato este que implica na importância de otimizar a rota de veículos para alcance deste último desafio mencionado.

Dentre as diversas ferramentas logísticas, segundo Gomes et al. (2012), a roteirização merece destaque, pois possibilita elaborar uma rota otimizada como objetivo de reduzir a distância percorrida e o tempo de transporte. Ainda segundo o autor, as atividades de transporte representam a maior parcela dos custos logísticos de uma empresa, por conseguinte, a roteirização torna-se uma estratégia para encontrar uma solução ótima e consequentemente uma redução dos custos.

Portanto, observa-se a importância da adoção de um modelo de roteirização e os vários modelos apresentados na literatura, recomendam a utilização de software. Amaral et al. (2014) afirmam que essa técnica mostra-se eficiente para otimizar o percurso, permitindo assim melhor precisão e menor tempo para a obtenção da rota. Desse modo, sua aplicação promove um serviço de alto nível aos clientes, além de reduzir os custos operacionais envolvidos, propiciando melhorias significativas para a empresa.

O presente estudo objetiva otimizar a rota de veículos da logística de distribuição de uma distribuidora de limpezas. Visando atingir melhorias no sistema logístico de distribuição por intermédio da otimização. 


\section{REFERENCIAL TEÓRICO}

\subsection{Transporte e Distribuição Física}

Para compreender os conceitos e atividades relacionadas ao transporte e distribuição física, a priori faz-se necessário observar sua relação aos canais de distribuição. Segundo Novaes (2015), a estratégia competitiva adotada pela empresa determina um esquema de distribuição específico e a partir dessa estrutura, são definidas as atividades logísticas relacionadas a distribuição física.

Sob esse enfoque, Carvalho (2009) afirma que o canal de distribuição corresponde ao conjunto de organizações responsáveis pela transferência do produto por toda cadeia de suprimento. Ainda segundo o supracitado, o canal de distribuição de um determinado produto pode envolver os seguintes setores: fabricante, atacadista, varejo e os serviços pós-venda.

Por isso, Santos (2014) afirma que a estruturação do canal de distribuição deve ser planejada adequadamente, visto que são estruturas rígidas, geralmente contratuais e, principalmente, em virtude dos riscos de perda em vendas devido a uma mudança mal elaborada.

Embora o termo seja recente, a essência das redes de distribuição física originou-se durante o período da primeira revolução industrial, impulsionada pela invenção da máquina a vapor e a substituição da madeira pelo aço que resultaram no barateamento dos custos de transporte e do aumento das embarcações (ROSA ${ }^{1}$, 2007). Desde então, conforme Carlos e Gordon (1986) as redes de distribuição configuram-se mais complexas e abrangentes, na mesma linha de raciocínio Novaes (2015) acrescenta que a distribuição física também engloba os processos operacionais e de controle, que permitem transferir os produtos desde o ponto de fabricação até o ponto de entrega ao consumidor.

Neste contexto, vários autores (DORNIER, 2012; SKWARA, 2013; RUSHTON et al., 2014), definem que o gerenciamento da distribuição física se dá em três níveis: estratégico, tático e operacional. Enomoto (2005), conceitua as atividades logísticas relativas a distribuição física em cada nível da seguinte forma:

- Nível Estratégico: Nesse nível ocorre a definição da rede logística, analisando aspectos como número e a localização de instalações 
produtivas e de armazenamento, assim como a escolha dos canais de distribuição, os meios de transporte e ainda os tipos de veículo mais adequado;

- Nível tático: Visa garantir eficiência nas operações do sistema de distribuição, definidas no nível estratégico. Dentre várias, destacam-se: o planejamento de transportes, escolha dos transportadores, além da análise da quantidade de veículos e as suas diferentes capacidades;

- Nível operacional: responsável pela programação, execução e controle das atividades rotineiras, visando assegurar o deslocamento dos produtos para os canais de distribuição ou para os mercados consumidores. Diversas atividades compõem a rotina da operação de distribuição, são exemplos: os procedimentos de armazenamento e movimentação interna de materiais, o processo de carga e descarga dos veículos, emissão de documentos e a programação dos roteiros de entregas.

Desta forma, o trabalho em questão dará suporte e busca melhorar de forma eficiente a atividade de transporte da distribuição física dos produtos da empresa em estudo. Portanto, pode-se considerar que o seguinte estudo está situado no nível operacional, uma vez que as atividades relacionadas a roteirização encontra-se nas etapas finais do processo de comercialização dos produtos: a de entrega das mercadorias (SANTOS, 2014).

Por consequência, atualmente, o estudo do transporte de cargas tornou-se sistêmico e de natureza científica, objetivando entender e analisar todas as variáveis envolvidas para melhor atender às complexas necessidades decorrentes das transações comerciais locais, regionais e globais (MARTINS; VICENTE, 2015). Assim, as decisões de transportes em logística envolvem a escolha dos diferentes modais, que deve-se considerar a relação necessária de tempo, distância, cursos, capacidade de carga (JACOBS; CHASE, 2012; CHOPRA; MEINDL, 2011).

Ainda no que diz respeito ao transporte de cargas, de acordo com Menchik (2010), os modais de transporte são importantes devido a seu serviço direto aos seus usuários, dentre os vários diferentes tipos de modais encontrados na literatura, Davidsson et al. (2005) os classificam da seguinte forma: aeroviário, dutoviário, ferroviário, hidroviário e rodoviário. 
Tendo em vista o supracitado por Gomes et al. (2012) na seção introdutória, a otimização das rotas tem por objetivo reduzir os custos logísticos e ao mesmo passo que de melhorar o nível de serviço. Desta forma, a seção seguinte trata dessa concepção de custo e nível de serviço.

\subsection{Custo Logístico e Nível de Serviço}

O custo logístico, está diretamente associado a execução efetiva de um serviço, assim segundo Martins e Vicente (2015) quando, por exemplo, são trocados os pneus de um caminhão, após o gasto requerido para a compra do mesmo, começa a incorrer um custo ao decorrer de sua utilização. Enquanto que o nível de serviço, ainda segundo o autor, é uma medida de qualidade, formado por aspectos quantitativos e característicos da qualidade do serviço prestado.

Nesse sentido Rosa ${ }^{2}$ (2011), define o nível de serviço como a percepção da qualidade, na ótica do cliente, ou seja, a organização deve estar atenta ao seu nicho de mercado e avaliar quais as variáveis como, por exemplo, prazo, confiabilidade, integridade da carga, atendimento ou o preço, são relevantes ao público alvo. Dessa forma, o autor afirma que o nível de serviço deve ser estabelecido e numericamente mensurável, não deixando margem à questionamentos, pois é um fator determinante para a satisfação dos consumidores e ainda, entender o quanto os mesmos estão dispostos a pagar pelo serviço.

Assim, entende-se que a integração das estratégias de serviço ao cliente, de localização e de transporte estão diretamente ligadas, visando garantir para a empresa maior eficiência nos processos, a redução os custos operacionais e, assim, gerando lucratividade para a empresa (LUIS,2009). Porém, as organizações possuem grandes dificuldades para mensurar era tear seus custos logísticos, conforme Rosa ${ }^{1}$ (2007), isso ocorre devido à falta de informações sobre custos, muitas vezes a grupados de forma ampla e agregada, dificultando uma análise mais detalhada.

A Classificação dos principais custos no âmbito logístico, segundo Martins e Vicente (2015), é dada da seguinte forma: 
- Custos diretos: São os custos associados diretamente ao produto ou serviço desde que haja uma medida de consumo, como a mão de obra, embalagem e outros envolvidos diretamente na prestação do serviço;

- Custos indiretos: Caracterizados por não poderem ser apropriados diretamente a cada tipo de objeto, produto ou serviço, como por exemplo, os custos com a tecnologia de informação utilizada em um processo logístico que atenda diversos clientes;

- Custos fixos: São custos associados ao funcionamento da organização, tais como o aluguel de um galpão para estocagem de produtos, que não há variação ao volume armazenado, por exemplo;

- Custos variáveis: Aqueles proporcionais a produção ou prestação de serviços, dentre vários pode-se citar o volume transportado, armazenado e dos serviços prestados.

Além disso, segundo Rosa ${ }^{1}$ (2007) os processos básicos de logística apresentam de forma agrupada as atividades necessárias para alcançar o objetivo da organização, essas atividades podem ser expressas na Equação1.

$\mathrm{C}_{T L}=\mathrm{Cl}_{I}+\mathrm{C}_{L}+\mathrm{C}_{\mathrm{PPI}}+\mathrm{C}_{\mathrm{A}}+\mathrm{C}_{\mathrm{T}}$

Onde:

$\mathrm{CTL}=$ Custo Total das Atividades de Logística;

$\mathrm{Cl}=$ Custo de Inventário;

$\mathrm{CL}=$ Custo de Lote;

$\mathrm{CPPI}=$ Custo de Processamento de Pedidos e Informação;

$\mathrm{CA}=$ Custo de Armazenagem;

$\mathrm{CT}=$ Custo de Transporte;

Assim, segundo Rosa' (2007, p. 34) "os custos totais correspondem a somados custos de transporte, custos de armazenagem, custos de processamento e informações de pedidos, custos associados ao tamanho do lote e custos de manutenção de estoque". Além disso, Yasar (2017) ressalta que os custos logísticos aumentam consideravelmente quando há deficiências existentes na integração entre os sistemas de transporte existentes, logo, para uma redução dos custos totais faz- 
se necessário uma eficiencia na integração e no uso dos veículos em uma organização.

Kazimírová et al. (2015) acrescentam ainda que os custos de logística incluem itens que ao calcular custos de produção, permanecem ocultos, ou seja, custos que não são contabilizados. Dessa forma, é importante o processo de monitoramento e registro de custo logístico, definindo quais custos dos processos corporativos estão incluídos nos custos logísticos e especificar o ponto de contato com o resto das unidades, por exemplo, a aquisição, produção, vendas, entre outros. Ainda segundo os autores, é necessário selecionar parâmetros adequados para expressar saídas logísticas, considerando cuidadosamente as condições específicas da empresa.

Dessa forma, segundo o Instituto de Logística e Supply Chain (ILOS, 2017) os custos logísticos associados ao transporte se classificam em custos fixos e variáveis. Os custos fixos representam os custos que ocorrem de maneira independente ao deslocamento do caminhão, como por exemplo, os funcionários (motorista), custos administrativos, seguro do veículo, IPVA/seguro obrigatório, dentre outros. Enquanto os custos que variam de acordo com a distância percorrida são considerados variáveis, descritos conforme a Equação (2).

$\mathrm{Cv}=C_{\text {comb }}+C_{\text {ôleo }}+C_{\text {lav } / \text { lub }}+C_{\text {pneus }}+C_{\text {manu }}+C_{\text {ped }}$

Custo com combustível: $C_{\text {comb }}=\frac{\text { Preço por litro }}{\text { Rendimento }}$

Custo com trocas de óleo: $C_{\text {óleo }}=\frac{\text { Preço por litro } x \text { capacidade do tanque }}{\text { Intervalo entre as trocas }}$

Custo com lavagem/lubrificação: $C_{\text {lav/lub }}=\frac{\text { custo da lavagem/lubrificaç̧̃ิo }}{\text { Intervalo }(\mathrm{Km})}$

Custo com Pneus: $C_{P_{\text {neus }}}=\frac{Q \text { de de Pneus } x\left(P_{1} x Q \text { de de recapagens } x P_{2}\right)}{\text { Vida útil de um Pneu com recapagem }}$

Custo com manutenção: $C_{\text {manutençẫo }}=\frac{\text { custo com manutençẫo total }}{\text { Quilometragem rodada }}$

Custos com pedágio: $C_{\text {pedágio }}=\frac{\text { custo de pedăgio }}{\text { Quilometragem rodada }}$ 
Neste sentido, pode-se concluir que realizações de todas as atividades logísticas devem criar um processo de sinergia, para que o resultado final seja garantir que o serviço será executado por um preço menor, e ainda, garantir o nível de serviço exigido (BOWERSOX et al, 2002). Por isso, a utilização de ferramentas logísticas como a roteirização, possibilitam 0 melhor uso dos recursos da organização, e consequentemente, é uma grande aliada para a redução nos custos operacionais e melhoria o nível de serviço prestado ao cliente.

\subsection{Método de Roteirização}

Segundo Ballou (2006) quando o processo de distribuição é realizado por uma firma terceiriza da a preocupação é direcionada ao valor dos fretes e da documentação requerida. Contudo, quando é realizado pela frota da própria organização interessa-se, especialmente, em programar os veículos de modo a alcançar eficiência no transporte, minimizando os custos operacionais e ainda garantir o nível de serviço desejado. Nesse contexto, estão situados os métodos de roteirização, objetivando otimizar os processos logísticos de distribuição em uma empresa.

Assim, pode-se conceituar a roteirização como o processo para a determinação dos roteiros ou sequência de paradas a serem realizadas pelos veículos da frota por meio de pontos geograficamente dispersos, em locais pré determinados para atendimento (CUNHA,2000).

De acordo com Enomoto (2005, p. 35), com relação ao tipo de modelagem, o problema da roteirização de veículos pode ser dividido em duas classes básicas:

- Problemas de roteirização em nós (NRP - Node Routing Problems), nos quais os locais de atendimento são representados por pontos específicos, caracterizados como nós;

- Problemas de roteirização em arcos (ARP - Arc Routing Problems), nos quais os locais de atendimento são representados de forma contínua ao longo de uma sequência de vias, caracterizados como arcos ou arestas.

Nesse contexto, o autor ainda afirma que os problemas de roteirização consideram as restrições geográficas e espaciais, existindo basicamente três grupos e/ou modelos, a saber: 
- Problema de roteirização pura: São considerados problemas espaciais que não consideram as variáveis temporais ou precedências entre as atividades para elaboração dos roteiros de coletas e/ou entrega;

- Problema de programação de veículos e tripulações: A problemática envolve a sequência das atividades para os veículos e com a movimentação da tripulação no espaço e no tempo;

- Problema de roteirização e programação: Considera os aspectos temporais dos roteiros, ou seja, as prioridades para o atendimento ou cumprimento de um horário.

Neto e Lima (2006) complementam que quando existe a ocorrência de aplicações com restrições de janelas de tempo e de precedência de tarefas, quando a coleta deve preceder a entrega e ambas devem estar alocadas ao mesmo veículo, a problemática envolvida envolve a combinação de roteirização e da programação de veículos.

No primeiro momento, conforme Melo e Ferreira Filho (2001) a primeira geração dos sistemas de roteirização e programação de veículos usavam os chamados mainframes, nos quais os resultados gerados nem sempre se disponibilizavam imediatamente, pois dependiam do tempo de processamento e da prioridade na fila de espera para resolução. Dessa forma, conforme os autores apenas por volta da década de noventa, em virtude do avanço tecnológico, e das intensas pesquisas desenvolvidas na área de pesquisa operacional, atualmente, os softwares possuem recursos computacionais, matemáticos e gráficos que proporcionam plataformas mais completas, voltadas a gestão da organização.

Por isso, Gomes (2012) afirma que a demanda por softwares de roteirização tem aumentado muito nos últimos anos, especialmente no Brasil. Dentre vários fatores, pode-se citar a estabilização da economia, as exigências por parte dos clientes, relacionadas a prazos, datas e horários de entrega, problemas de trânsito, acesso e circulação, a busca por um melhor atendimento, redução dos estoques e aumento na frequência das entregas. $O$ conjunto desses fatores tem feito com que várias empresas busquem tecnologias com a finalidade de melhorar em seus sistemas de roteirização.

Outro fator importante segundo os autores Cunha (2000) e Ballou (2006) consiste na escolha do software mais adequado à organização, uma vez que Revista Produção Online. Florianópolis, SC, v. 20, n. 2, p. 598-626, 2020 
condicionantes locais e as particularidades da empresa devem ser considerados, bem como a atenção as bases de dados referenciados que, nem sempre, estão devidamente atualizadas. A partir dessa análise, a empresa deve optar pelo software que proporcione o melhor custo-benefício, visto que atualmente, existem variadas opções no mercado, que requerem diferentes valores de investimento e manutenção (NOVAES, 2015).

Com as devidas ressalvas compreendidas, e considerando a realidade da empresa em estudo, optou-se por utilizar a ferramenta LOGWARE, que conforme Ballou (1999), o LOGWARE é um conjunto de programas para a análise e resolução de problemas logísticos. De acordo com Pereira et al (2015) complementa que o LOGWARE é um conjunto de programas que são importantes para a análise de uma variedade de problemas e estudos de caso envolvendo logística.

Ainda conforme o autor, os módulos encontrados no LOGWARE são: FORECAST; ROUTE; ROUTESEQ; ROUTER; INPOL; COG; MULTICOG; PMED; WARELOCA; LAYOUT; MILES; TRANLP; LNPROG; MIPROG; MULREG.

De acordo Ballou (1999), a função ROUTESEQ consiste em software heurístico apropriado para a resolução do problema do caixeiro viajante, uma vez que o mesmo traça uma sequência de até vinte paradas em uma rota mais um ponto de origem. O Autor ainda ressalta que cada parada e o ponto de origem são identificados por coordenadas lineares, na qual as distâncias euclidianas da linha reta são computadas em termos destas coordenadas. Dessa forma, o ROTSEQ apresenta-se como o módulo mais adequado a ser utilizado nessa pesquisa, visto que o mesmo determina a melhor sequência de rotas para otimizar a rota de veículos.

\section{PROCEDIMENTOS METODOLOGICOS}

\subsection{Coleta de Dados}

\subsubsection{Variáveis e indicadores}

Com intuito de alcançar os objetivos propostos nessa pesquisa, considerando 
também as informações requeridas para o uso do Software LOGWARE, as principais variáveis e indicadores considerados podem ser descritos conforme o Quadro 1.

Quadro 1 - Descrições das variáveis e indicadores
\begin{tabular}{|l|l|}
\hline \multicolumn{1}{|c|}{ VARIÁVEIS } & \multicolumn{1}{c|}{ INDICADORES } \\
\hline Demanda & Quantidade de pedidos \\
\hline Composição da frota de veículos & Quantidade (Número) e Capacidade (Área) \\
\hline $\begin{array}{l}\text { Quadro de Funcionários/ motoristas } \\
\text { envolvidos }\end{array}$ & Quantidade (Número) \\
\hline Endereço dos Clientes & Coordenadas geográficas \\
\hline Tempo entre o pedido e recebimento & Tempo (h) \\
\hline
\end{tabular}

Fonte: Elaborado pelos autores

Além das variáveis e indicadores presentes no Quadro 1, também levantou-se as principais restrições relativas ao processo de atendimento, bem como informações referentes aos produtos e as estratégias de atendimento adotada pela organização estudada.

\subsubsection{Instrumentos de coleta de dados}

No que se referem aos instrumentos dados coletados, os mesmos foram obtidos a partir do MRP da empresa, além de informações técnicas como a composição da frota de veículos, endereço dos clientes, quadro de funcionários dentre outras informações fornecidas pela empresa.

Além disso, as demais informações referentes ao tempo médio de carga e descarga e os procedimentos de atendimento aos clientes, por exemplo, foram medidos diretamente com o uso de cronômetro digital, além de entrevistas não estruturadas com o gestor responsável e os motoristas da empresa e da observação sistemática. 


\subsubsection{Procedimentos para coleta de dados}

Uma vez definido as variáveis e os instrumentos para a coleta de dados, nesse tópico será apresentado os procedimentos para a coleta de dados. Assim, para a coleta dados foram realizadas visitas in loco na distribuidora de forma a obter as informações necessárias ao processo de distribuição de cargas.

As visitas foram realizadas no período de 18 dias, de segunda-feira à sextafeira, uma vez que a organização não funciona aos finais de semana. Também, é válido ressaltar que não se utilizou o processo de amostragem para selecionar as rotas, pois por conveniência utilizou a própria população de veículos de transporte da empresa, que se trata de 4 motocicletas. Além disso, as visitas ocorreram no turno da tarde da organização, pois, segundo o gestor, é quando há maior frequência de despacho de pedidos aos consumidores.

As visitas inicialmente objetivaram levantar informações mais específicas do processo de distribuição da organização, tais como o número e a capacidade dos veículos, o quadro de motoristas disponíveis, será observado e calculado o tempo médio para carga e descarga dos produtos, além de elencar as principais restrições presentes na distribuição dos produtos da organização. Posteriormente, por meio do acesso ao sistema de informação da empresa, obtiveram-se os dados referente a localização dos clientes.

Essas e as demais informações foram listadas com o auxílio do software Microsoft Excel $\circledast$ (Apêndice A), para assim estimar o cálculo dos custos logísticos de distribuição que a organização possui com o método atual. Finalmente, com os devidos dados obtidos, iniciou-se o processo de tratamento e análise dos mesmos que será exposto no próximo tópico.

\subsection{Procedimentos de Tratamento e Análise de Dados}

Tendo os dados coletados em mãos estes foram tratados e analisados, tendo em vista o alcance dos resultados propostos nos objetivos específicos desta pesquisa. Em primeira instância, foram tabulados e apreciados para, posteriormente, foram importados para o software LOGWARE. 
Desta forma, elaborou-se uma proposição de novas rotas de distribuição de produtos com intuito de otimizar as já existentes na empresa. Posteriormente, avaliou-se a proposição por meio de indicadores de desempenho logísticos em uma planilha eletrônica estruturada no Microsoft Excel ® como visto no Apêndice B. Esses indicadores permitiram quantificar os ganhos por meio de uma análise comparativa entre o método atual e o otimizado, evidenciando os impactos e/ou benefícios que a adoção do modelo proposto proporcionou a organização.

\section{RESULTADOS}

\subsection{Análise das Rotas Atuais}

Á medida que os clientes efetuam os pedidos, foram levantados dados referentes a sua localização, contendo as coordenadas geográficas dos estabelecimentos obtidos a partir do sistema de ERP da organização. Para fins de facilitar a explicação dos calculos, considerando um grande volume de tabelas geradas no período analisado, optou-se por usar o primeiro dia de coleta de dados como exemplo. No entanto sabe-se que o período total foi de 18 dias como explanado na metodologia. A Tabela 1 contém as rotas dos quatro veículos e a localizações geográficas dos clientes atendido na data de exemplo.

Observando a Tabela 1, nota-se que na referente data houveram 17 entregas realizadas por todos os 4 veículos (Xi para $X=$ a letra que representa um cliente e i = ao número do veículo), sendo que cada veículo atendeu de três a seis clientes por rota, essa variação se deve à relação entre a capacidade do veículo, o volume e a ordem dos pedidos. Além disso, é valido ressaltar que os motoristas fazem uma rota por turno de trabalho, exceto em situações específicas quando o tempo necessário para o atendimento da rota excede esse período. Nas demais datas são observados o mesmo padrão, uma vez que os motoristas realizam as rotas de maneira intuitiva, de acordo com a demanda dos pedidos. 
Tabela 3 - Localizações geográficas dos clientes no dia 14/08/2017

\begin{tabular}{|c|c|c|c|c|c|c|c|c|}
\hline \multicolumn{9}{|c|}{ Veículo 1} \\
\hline \multirow[b]{2}{*}{ Clientes } & \multicolumn{4}{|c|}{ X (Latitude) } & \multicolumn{4}{|c|}{ Y (Longitude) } \\
\hline & Graus & Min & Seg & $\begin{array}{c}\text { Valor } \\
\text { Absoluto }\end{array}$ & Graus & Min & Seg & $\begin{array}{c}\text { Valor } \\
\text { Absoluto }\end{array}$ \\
\hline Armazém & 5 & 4 & 26,01 & 5,07389 & 42 & 47 & 1,19 & 42,7836 \\
\hline A1 & 5 & 6 & 23,24 & 5,10645 & 42 & 47 & 30,46 & 42,7917 \\
\hline B1 & 5 & 4 & 9,14 & 5,06920 & 42 & 47 & 33,25 & 42,7925 \\
\hline C1 & 5 & 4 & 12,98 & 5,07027 & 42 & 48 & 32,58 & 42,8090 \\
\hline D1 & 5 & 7 & 6,74 & 5,11853 & 42 & 47 & 51,12 & 42,7975 \\
\hline \multicolumn{9}{|c|}{ Veículo 2} \\
\hline \multirow[b]{2}{*}{ Clientes } & \multicolumn{4}{|c|}{ X (Latitude) } & \multicolumn{4}{|c|}{ Y (Longitude) } \\
\hline & Graus & Min & Seg & $\begin{array}{c}\text { Valor } \\
\text { Absoluto }\end{array}$ & Graus & Min & Seg & $\begin{array}{c}\text { Valor } \\
\text { Absoluto }\end{array}$ \\
\hline Armazém & 5 & 4 & 26,01 & 5,073891 & 42 & 47 & 1,19 & 42,8366 \\
\hline A2 & 5 & 6 & 18,09 & 5,105025 & 42 & 45 & 23,4 & 42,7565 \\
\hline B2 & 5 & 5 & 51,16 & 5,097544 & 42 & 44 & 14,14 & 42,7372 \\
\hline C2 & 5 & 9 & 10,32 & 5,152866 & 42 & 47 & 18,02 & 42,7883 \\
\hline \multicolumn{9}{|c|}{ Veículo 3} \\
\hline \multirow{3}{*}{ Clientes } & \multicolumn{4}{|c|}{ X (Latitude) } & \multicolumn{4}{|c|}{ Y (Longitude) } \\
\hline & \multirow[b]{2}{*}{ Graus } & & & \multirow{2}{*}{$\begin{array}{c}\text { Valor } \\
\text { Absoluto }\end{array}$} & \multirow[b]{2}{*}{ Graus } & \multirow[b]{2}{*}{ Min } & \multirow[b]{2}{*}{ Seg } & Valor \\
\hline & & Min & Seg & & & & & Absoluto \\
\hline Armazém & 5 & 4 & 26,01 & 5,073891 & 42 & 47 & 1,19 & 42,7836 \\
\hline A3 & 5 & 6 & 23,43 & 5,106508 & 42 & 48 & 31,88 & 42,8088 \\
\hline B3 & 5 & 5 & 56,91 & 5,099141 & 42 & 49 & 12,37 & 42,8201 \\
\hline C3 & 5 & 4 & 38,58 & 5,077383 & 42 & 49 & 5,46 & 42,8181 \\
\hline D3 & 5 & 5 & 30,28 & 5,091744 & 42 & 48 & 4,85 & 42,8013 \\
\hline E3 & 5 & 4 & 24,95 & 5,073597 & 42 & 45 & 39,68 & 42,7610 \\
\hline F3 & 5 & 4 & 42,50 & 5,078472 & 42 & 46 & 26,36 & 42,7739 \\
\hline \multicolumn{9}{|c|}{ Veículo 4} \\
\hline \multirow{3}{*}{ Clientes } & \multicolumn{3}{|c|}{ X (Latitude) } & & \multicolumn{4}{|c|}{ Y (Longitude) } \\
\hline & & & & Valor & & & & Valor \\
\hline & Graus & Min & Seg & Absoluto & Graus & Min & Seg & Absoluto \\
\hline Armazém & 5 & 4 & 26,01 & 5,073891 & 42 & 47 & 1,19 & 42,7836 \\
\hline A4 & 5 & 6 & 25,16 & 5,106988 & 42 & 44 & 59,12 & 42,7497 \\
\hline B4 & 5 & 6 & 19,99 & 5,105552 & 42 & 45 & 42,87 & 42,7619 \\
\hline C4 & 5 & 3 & 0,12 & 5,050033 & 42 & 49 & 3,04 & 42,8175 \\
\hline D4 & 5 & 4 & 49,81 & 5,080502 & 42 & 46 & 52,57 & 42,7812 \\
\hline
\end{tabular}

Fonte: Elaborado pelos autores

Em seguida, logo durante chegada do veículo a distribuidora, é levantado as informações referentes a ordem das entregas dos pedidos (rota realizada), e dos indicadores de desempenho em relação a quilometragem percorrida e a medição do tempo de atendimento. Essas informações são ilustradas conforme a Tabela 2:

Os dados da Tabela 2 foram, posteriormente, usados para o comparativo com a rota otimizada proposta pelo software. No mapeamento da rota realizada pelo veículo 1 do dia 14/08/2017, na qual os pontos CDAB representam as ordens de paradas no processo de atendimento. 
Tabela 2 - Indicadores de desempenho da rota atual no dia 14/08/2017

\begin{tabular}{lccc}
\hline Veículos & Rota & Distância $\mathbf{( k m )}$ & $\begin{array}{c}\text { Tempo de At. Real } \\
\text { (h) }\end{array}$ \\
\hline Veículo 1 & CDAB & 14,7 & 2,4 \\
Veículo 2 & ABC & 22,0 & 3,5 \\
Veículo 3 & CEFBDA & 19,0 & 3,0 \\
Veículo 4 & CDAB & 19,3 & 3,1 \\
\hline & TOTAL & $\mathbf{7 5 , 0 4}$ & $\mathbf{1 2 , 0 0}$ \\
\hline
\end{tabular}

Fonte: Elaborado pelos autores

Além dos dados da Tabela 2, calcularam-se os custos envolvidos na distribuição dos produtos, é importante ressaltar que os cálculos foram realizados apenas considerando os custos variáveis, uma vez que, segundo o instituto ILOS (2017) os custos fixos representam os custos que ocorrem de maneira independente ao deslocamento, ou seja, serão de igual valor independente da rota realizada na distribuição dos produtos.

Dessa forma, calcularam-se os custos variáveis referentes ao período analisado, cujos dados foram baseados em informações fornecidas pelo fabricante dos veículos (manual do proprietário CG 125 Cargo), em conjuntura com informações repassadas pelo gestor da empresa, as variáveis referentes ao custeio variável podem ser consultadas no Quadro 2.

Quadro 2 - Informações importantes para o custeio variável

\begin{tabular}{|l|l|l|}
\hline \multicolumn{1}{|c|}{ Variáveis importantes } & \multicolumn{1}{c|}{ Valor } & \multicolumn{1}{c|}{ Custo } \\
\hline Rendimento (Consumo urubano-Gasolina) & $30(\mathrm{~km} / \mathrm{l})$ & $\mathrm{R} \$ 3,70$ \\
\hline $\begin{array}{l}\text { Troca de Óleo (Mobil super moto 4T } \\
\text { multiviscoso SAE 20W-50 API-SF) }\end{array}$ & $1500 \mathrm{~km}$ & $\mathrm{R} \$ 15,90$ \\
\hline Vida útil do Pneu (Pirelli) & $20000 \mathrm{~km}$ & $\mathrm{R} \$ 90,00$ \\
\hline Manutenção & $10000 \mathrm{~km}$ & $\mathrm{R} \$ 335,00$ \\
\hline
\end{tabular}

Fonte: Elaborado pelo autor

É importante salientar que os valores referentes ao rendimento, embora o fabricante forneça o valor de $40 \mathrm{~km} / \mathrm{l}$, segundo o gestor devido ao uso da motocicleta para transporte de carga, o valor do rendimento reduz para aproximadamente $30 \mathrm{~km} / \mathrm{l}$ (Quadro 2), este fato deve-se ao compartimento (carroceria) acoplado a motocicleta para transportar as cargas. Nesse sentido, o mesmo ocorre com a vida útil de um pneu, o fabricante recomenda a troca após $25000 \mathrm{~km}$ rodados, porém esse valor 
reduz para $20000 \mathrm{~km}$ em virtude do peso adicional às motocicletas durante as entregas.

Além disso, para o cálculo do custo com a manutenção consideraram-se valores monetários obtidos mediante a consulta de documentos referentes as manutenções anteriores realizadas pela empresa.

Posteriormente, somaram-se as distâncias percorridas dos quatro veículos ao final de cada dia durante o período total analisado, esses valores podem ser visualizado no Quadro 3.

Quadro 3 - Distância percorrida no período analis
\begin{tabular}{|l|l|}
\hline \multicolumn{1}{|c|}{ Dias } & \multicolumn{1}{c|}{ Rota Atual } \\
\hline $1^{\circ}$ dia & $75,04 \mathrm{~km}$ \\
\hline $2^{\circ}$ dia & $76,86 \mathrm{~km}$ \\
\hline $3^{\circ}$ dia & $86,12 \mathrm{~km}$ \\
\hline $4^{\circ}$ dia & $65,6 \mathrm{~km}$ \\
\hline $5^{\circ}$ dia & $93,55 \mathrm{~km}$ \\
\hline $6^{\circ}$ dia & $60,38 \mathrm{~km}$ \\
\hline $7^{\circ}$ dia & $75,79 \mathrm{~km}$ \\
\hline $8^{\circ}$ dia & $66,94 \mathrm{~km}$ \\
\hline $9^{\circ}$ dia & $82,74 \mathrm{~km}$ \\
\hline $10^{\circ}$ dia & $67,28 \mathrm{~km}$ \\
\hline $11^{\circ}$ dia & $87,01 \mathrm{~km}$ \\
\hline $12^{\circ}$ dia & $99,53 \mathrm{~km}$ \\
\hline $13^{\circ}$ dia & $75,79 \mathrm{~km}$ \\
\hline $14^{\circ}$ dia & $88,51 \mathrm{~km}$ \\
\hline $15^{\circ}$ dia & $85,5 \mathrm{~km}$ \\
\hline $16^{\circ}$ dia & $92,06 \mathrm{~km}$ \\
\hline $17^{\circ}$ dia & $109,24 \mathrm{~km}$ \\
\hline $18^{\circ}$ dia & $99,41 \mathrm{~km}$ \\
\hline $\mathrm{TO}^{\circ} \mathrm{TAL}$ & $1487,3 \mathrm{~km}$ \\
\hline Fon $\mathrm{E}$ & \\
\hline
\end{tabular}

Fonte: Elaborado pelos autores

Finalmente, após a obtenção das informações referidas nos Quadros 2 e 3, calculou-se os custos variáveis do período total analisado, cujo valores se encontram na Tabela 3. 
Tabela 3 - Custos variáveis da distribuição atual no período total analisado

\begin{tabular}{|c|c|c|c|}
\hline Custos & & lor & $\begin{array}{l}\text { Percentual de } \\
\text { participação }\end{array}$ \\
\hline Custos com combustível & $\mathrm{R} \$$ & 183,43 & $69,94 \%$ \\
\hline Custos com troca de óleo & $\mathrm{R} \$$ & 15,86 & $6,06 \%$ \\
\hline Custos com pneus & $\mathrm{R} \$$ & 13,39 & $5,10 \%$ \\
\hline Custos com manutenção & $\mathrm{R} \$$ & 49,58 & $18,90 \%$ \\
\hline Custos com pedágio & & - & - \\
\hline Custos com lavagem & & - & - \\
\hline TOTAL & $\mathbf{R} \$$ & 262,26 & $100 \%$ \\
\hline
\end{tabular}

Fonte: Elaborado pelos autores

A partir da Tabela 3, observa-se que no modelo de distribuição atual, durante o período total analisado, correspondente ou turno vespertino nas quatro semanas, o valor dos custos variáveis é de 262,26 Reais. Outro fator importante analisado é que a organização não possui custos com pedágio e lavagem dos veículos. Além disso, o custo com combustível possui o maior impacto nesse montante, representando isoladamente cerca de $69,94 \%$ dos custos variáveis totais. Este último fato reforça que a melhoria nas rotas impacta na redução significante dos custos já que o combustível é um custo variável e que depende da distância percorrida.

\subsection{Elaboração e Análise das Rotas Otimizadas}

Para a otimização de rotas, foi utilizado o software LOGWARE, especificamente a função ROUTSEQ, definida como a função do Caixeiro Viajante, que visa elaborar uma sequência de cidades a serem visitadas por um caixeiro viajante de forma a diminuir as distâncias passando por uma localidade uma única vez (CUNHA, 2000). Porém, o software possui algumas especificações e limitações, dessa forma, para a correta utilização inicialmente foi definida um local como ponto zero, para auxiliar nos cálculos de coordenadas relativas.

Nesse contexto, além do uso das coordenadas do armazém e dos clientes para o cálculo das rotas, escolheu-se um marco da cidade onde a empresa está localizada (Praça da Liberdade), como ponto de referência para efetuar os cálculos no software, pois dessa forma o armazém ficava disposto no quadrante positivo. Para melhor assimilação das informações a Tabela 4, evidencia os cálculos 
realizados referente a mesma data do tópico anterior, correspondente ao primeiro dia analisado.

Tabela 4 - Matriz coordenadas relativas no dia 14/08/2017

\begin{tabular}{|c|c|c|c|c|}
\hline \multicolumn{5}{|c|}{ Veículo 1} \\
\hline \multirow{2}{*}{ Clientes } & \multicolumn{2}{|c|}{ X (Latitude) } & \multicolumn{2}{|c|}{ Y (Longitude) } \\
\hline & X ABS & X Relativo & Y ABS & Y Relativo \\
\hline Praça da Liberdade & 5,09033333 & 0 & 42,8109833 & 0 \\
\hline Armazém & 5,07389167 & 0,016 & 42,7836639 & 0,027 \\
\hline A & 5,10645556 & $-0,016$ & 42,7917944 & 0,019 \\
\hline$B$ & 5,06920556 & 0,021 & 42,7925694 & 0,018 \\
\hline C & 5,07027222 & 0,020 & 42,80905 & 0,002 \\
\hline D & 5,11853889 & $-0,028$ & 42,7975333 & 0,013 \\
\hline \multicolumn{5}{|c|}{ Veículo 2} \\
\hline \multirow{2}{*}{ Clientes } & \multicolumn{2}{|c|}{ X (Latitude) } & \multicolumn{2}{|c|}{ Y (Longitude) } \\
\hline & X ABS & X Relativo & Y ABS & Y Relativo \\
\hline Praça da Liberdade & 5,09033333 & 0 & 42,8109833 & 0 \\
\hline Armazém & 5,07389167 & 0,016 & 42,7836639 & 0,027 \\
\hline A & 5,105025 & $-0,015$ & 42,7565 & 0,054 \\
\hline$B$ & 5,09754444 & $-0,007$ & 42,7372611 & 0,074 \\
\hline C & 5,15286667 & $-0,063$ & 42,7883389 & 0,023 \\
\hline \multicolumn{5}{|c|}{ Veículo 3} \\
\hline \multirow{2}{*}{ Clientes } & \multicolumn{2}{|c|}{ X (Latitude) } & \multicolumn{2}{|c|}{ Y (Longitude) } \\
\hline & X ABS & X Relativo & Y ABS & Y Relativo \\
\hline Praça da Liberdade & 5,09033333 & 0 & 42,8109833 & 0 \\
\hline Armazém & 5,07389167 & 0,016 & 42,7836639 & 0,027 \\
\hline A & 5,10650833 & $-0,016$ & 42,8088556 & 0,002 \\
\hline B & 5,09914167 & $-0,009$ & 42,8201028 & $-0,009$ \\
\hline C & 5,07738333 & 0,013 & 42,8181833 & $-0,007$ \\
\hline D & 5,09174444 & $-0,001$ & 42,8013472 & 0,010 \\
\hline$E$ & 5,07359722 & 0,017 & 42,7610222 & 0,050 \\
\hline$F$ & 5,07847222 & 0,012 & 42,7739889 & 0,037 \\
\hline \multicolumn{5}{|c|}{ Veículo 4} \\
\hline \multirow{2}{*}{ Clientes } & \multicolumn{2}{|c|}{ X (Latitude) } & \multicolumn{2}{|c|}{ Y (Longitude) } \\
\hline & X ABS & X Relativo & Y ABS & Y Relativo \\
\hline Praça da Liberdade & 5,09033333 & 0 & 42,8109833 & 0 \\
\hline Armazém & 5,07389167 & 0,016 & 42,7836639 & 0,027 \\
\hline A & 5,10698889 & $-0,017$ & 42,7497556 & 0,061 \\
\hline$B$ & 5,10555278 & $-0,015$ & 42,7619083 & 0,049 \\
\hline C & 5,05003333 & 0,040 & 42,8175111 & $-0,007$ \\
\hline D & 5,08050278 & 0,010 & 42,7812694 & 0,030 \\
\hline
\end{tabular}

Fonte: Elaborado pelos autores 
Uma vez convertidas para coordenadas cartesianas, é possível inserir os dados no software. Para o uso desta ferramenta foi considerado o fator de escala $1: 100$, sendo as coordenadas cartesianas da distribuidora no valor de $0,016 \mathrm{x}$ e $0,027 y$. Concluindo todos os passos descritos, foi possível calcular a rota ótima para cada veículo. Após o preenchimento e selecionando a opção "Solve", o software fornece a melhor ordem das paradas e indica a distância a ser percorrida.

Dessa forma, observa-se que o software fornece a ordem das paradas e ainda a distância total da rota, nesse caso encontra-se no valor de 12,197 km. No mapeamento da rota otimizada pelo software na qual os pontos BCDE representam as ordens de paradas no processo de atendimento, os pontos $\mathrm{A}$ e $\mathrm{F}$ refere-se ao ponto inicial e final da rota, ou seja, o armazém da distribuidora.

Ao analisarmos os dados coletados, observa-se um sequenciamento mais uniforme entre as paradas, e consequentemente, uma maior eficiência no deslocamento. Os cálculos foram efetuados a todas as rotas realizadas pelas quatro motocicletas no período analisado, para exemplificar a Tabela 5 com as informações referentes as rotas otimizadas, também é referente a data 14/08/2017.

Tabela 5 - Planilha com informações da rota otimizada no dia 14/08/2017

\begin{tabular}{|c|c|c|c|}
\hline LOGWARE & Rota & Distância (km) & Tempo de At. Real (h) \\
\hline Veículo 1 & DBCA & 11,7 & 2,3 \\
\hline Veículo 2 & BAC & 22,0 & 3,5 \\
\hline Veículo 3 & CEFDAB & 15,9 & 2,9 \\
\hline Veículo 4 & CDAB & 19,3 & 3,1 \\
\hline \multicolumn{2}{|c|}{ TOTAL } & 68,88 & 11,8 \\
\hline
\end{tabular}

Fonte: Elaborado pelos autores

Com as informações coletadas foram somadas as distâncias indicadas pelo software dos quatro veículos e somadas ao final de cada dia durante todo o período analisado, de acordo com o Quadro 4.

Finalmente, após a obtenção das informações referidas no Quadro 4 e das informações previamente estabelecidas no Quadro 2 da seção anterior, estimou-se os custos variáveis, cujo valores se encontram na Tabela 6. 
Quadro 4 - Distância indicada pelo software para o período analisado

\begin{tabular}{|c|c|}
\hline Dias & Rota Otimizada \\
\hline $1^{\circ}$ dia & $68,88 \mathrm{~km}$ \\
\hline $2^{\circ}$ dia & $72,46 \mathrm{~km}$ \\
\hline 3ㅇ dia & $73,1 \mathrm{~km}$ \\
\hline $4^{0} \mathrm{dia}$ & $62,18 \mathrm{~km}$ \\
\hline $5^{\circ} \mathrm{dia}$ & $73,66 \mathrm{~km}$ \\
\hline $6^{\circ} \mathrm{dia}$ & $53,46 \mathrm{~km}$ \\
\hline $7^{0} \mathrm{dia}$ & $66,01 \mathrm{~km}$ \\
\hline $8^{\circ}$ dia & $59,69 \mathrm{~km}$ \\
\hline 9 dia & $75,8 \mathrm{~km}$ \\
\hline $10^{\circ}$ dia & $56,77 \mathrm{~km}$ \\
\hline $11^{\circ}$ dia & $75,61 \mathrm{~km}$ \\
\hline $12^{\circ}$ dia & $77,91 \mathrm{~km}$ \\
\hline $13^{\circ}$ dia & $69,18 \mathrm{~km}$ \\
\hline $14^{\circ}$ dia & $75,64 \mathrm{~km}$ \\
\hline $15^{\circ}$ dia & $77,95 \mathrm{~km}$ \\
\hline $16^{\circ}$ dia & $78,74 \mathrm{~km}$ \\
\hline $17^{\circ}$ dia & $100,75 \mathrm{~km}$ \\
\hline $18^{\circ}$ dia & $86,05 \mathrm{~km}$ \\
\hline TOTAL & $1303,8 \mathrm{~km}$ \\
\hline
\end{tabular}

Fonte: Elaborado pelos autores

Tabela 6 - Estimativa dos custos variáveis de distribuição

\begin{tabular}{lrr}
\multicolumn{1}{c}{ Custos } & \multicolumn{2}{c}{ Valor } \\
\hline Custos com combustível & $\mathrm{R} \$$ & 160,80 \\
Custos com troca de óleo & $\mathrm{R} \$$ & 13,91 \\
Custos com pneus & $\mathrm{R} \$$ & 11,73 \\
Custos com manutenção & $\mathrm{R} \$$ & 43,46 \\
Custos com pedágio & & \multicolumn{2}{c}{-} \\
Custos com lavagem & & \multicolumn{2}{c}{-} \\
\hline
\end{tabular}

Fonte: Elaborado pelos autores

A partir da Tabela 6, nota-se que para as rotas indicadas pelo software a estimativa do valor dos custos variáveis é de 229,9 Reais. De igual maneira ao cálculo com as rotas atuais, não houve custos com pedágio e lavagem dos veículos, sendo o custo com combustível a variável de maior impacto, representando cerca de $70 \%$ dos custos variáveis totais. 


\subsection{Comparação e Análise dos Indicadores}

Com o objetivo de realizar a análise dos impactos e/ou benefícios que a roteirização otimizada por meio do LOGWARE proporcionaria para empresa em estudo, definiu-se alguns parâmetros, são eles: a distância percorrida, o tempo de atendimento e os custos envolvidos com a distribuição de produtos.

\subsubsection{Primeiro indicador: distância percorrida}

Para o cálculo da distância percorrida, foram somados os valores de deslocamento dos veículos para cada dia de coleta de dados, dados obtidos a partir das planilhas ilustradas conforme a Tabela 2. De maneira análoga, realizou-se com relação ao deslocamento indicado pelo software na rota otimizada. Os valores encontrados são ilustrados no Gráfico 1.

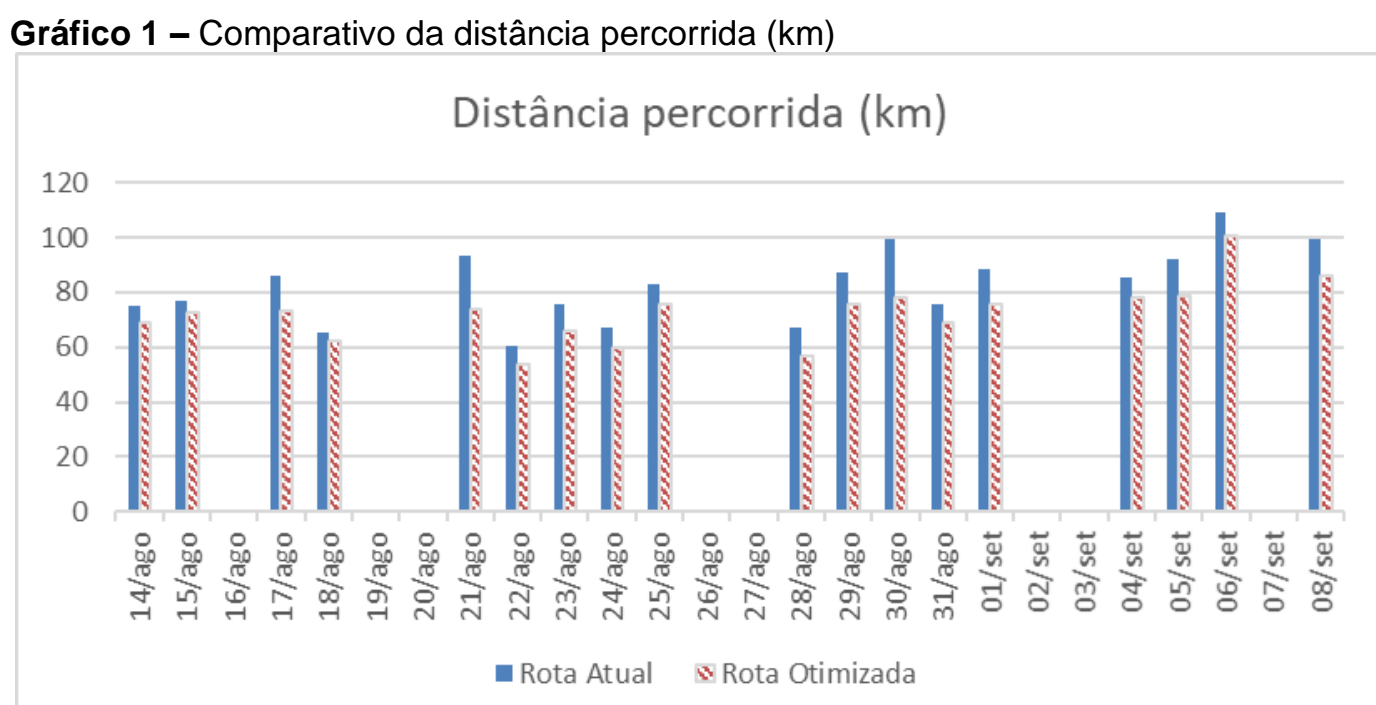

Fonte: Elaborado pelos autores

A partir do Gráfico 1, é notório que a rota otimizada pelo software é sempre de menor valor de deslocamento, essa diferença ainda se torna mais evidente em dias de distâncias mais elevadas, a Tabela 7 possui os dados desse indicador contendo o valor total de redução para o período analisado. 
Tabela 7 - Indicador: distância percorrida

\begin{tabular}{cccc}
\hline Indicador & Rota Atual & Rota Otimizada & Redução \\
\hline Distância percorrida & $1487,3 \mathrm{~km}$ & $1303,8 \mathrm{~km}$ & $183,5 \mathrm{~km}$
\end{tabular}

Fonte: Elaborado pelos autores

Analisando a Tabela 7, percebe-se que a rota otimizada possui uma redução no valor total de $183,5 \mathrm{~km}$ com relação a rota realizada, distância percorrida por aproximadamente dois dias normais de funcionamento da distribuidora. Essa diferença de deslocamento é relevante, uma vez que a distância percorrida impacta no valor dos custos variáveis e ainda no tempo de atendimento (nível de serviço), por isso, essa redução é um indicativo positivo, evidenciando os benefícios que o uso da roteirização proporcionaria para a organização em estudo.

\subsubsection{Segundo indicador: tempo de atendimento}

O segundo parâmetro a ser analisado diz respeito ao tempo de atendimento, para a medição dessa variável inicialmente foram coletados os dados referentes aos horários entre o horário de saída e chagada de cada veículo do armazém, conforme foi evidenciado na Tabela 2 da seção anterior. Em seguida, os dados referentes ao tempo de atendimento foram somados e contabilizados para cada dia durante toda a realização da coleta de dados, como ilustra o Quadro 4.

A partir dessas informações do Quadro 4 estimou-se o tempo de atendimento referente as rotas recomendadas pelo software. Os cálculos basearam-se apenas na variação do deslocamento, uma vez que as demais variáveis como o tempo de efetuar o pedido, separação dos produtos para distribuição, o faturamento, a carga e descarga das mercadorias, possuem tempos igualitários para os dois cenários analisados.

Dessa forma, subtraiu-se as distâncias entre a rota realizada e a rota otimizada, considerou-se também uma velocidade média de $30 \mathrm{~km} / \mathrm{h}$ no deslocamento, valor obtido por meio dos dados das entregas já realizadas, conforme o modelo da Tabela 2. Com as devidas considerações, estimou-se o tempo de atendimento, cujo comparativo pode ser observado no Gráfico 2. 
Quadro 4 - Tempo de atendimento diário (h)

\begin{tabular}{|c|c|}
\hline Dias & Rota Atual (h) \\
\hline 14/ago & $12 \mathrm{~h}$ \\
\hline 15/ago & $12,2 \mathrm{~h}$ \\
\hline 17/ago & $13,7 \mathrm{~h}$ \\
\hline 18/ago & $10,5 \mathrm{~h}$ \\
\hline 21/ago & $14,9 h$ \\
\hline 22/ago & $9,7 \mathrm{~h}$ \\
\hline 23/ago & $12 \mathrm{~h}$ \\
\hline 24/ago & $10,6 \mathrm{~h}$ \\
\hline 25/ago & $13,2 \mathrm{~h}$ \\
\hline 28/ago & $10,7 \mathrm{~h}$ \\
\hline 29/ago & $14 \mathrm{~h}$ \\
\hline 30/ago & $16 \mathrm{~h}$ \\
\hline 31/ago & $12,2 \mathrm{~h}$ \\
\hline $01 / \mathrm{set}$ & $14,2 h$ \\
\hline $04 /$ set & $13,7 \mathrm{~h}$ \\
\hline $05 /$ set & $14,5 \mathrm{~h}$ \\
\hline $06 /$ set & $17,5 h$ \\
\hline $08 /$ set & $15,8 \mathrm{~h}$ \\
\hline TOTAL & $237,4 \mathrm{~h}$ \\
\hline
\end{tabular}

Fonte: Elaborado pelos autores

Gráfico 2 - Comparativo entre os tempos de atendimento (h)

\section{Tempo de Atendimento (h)}

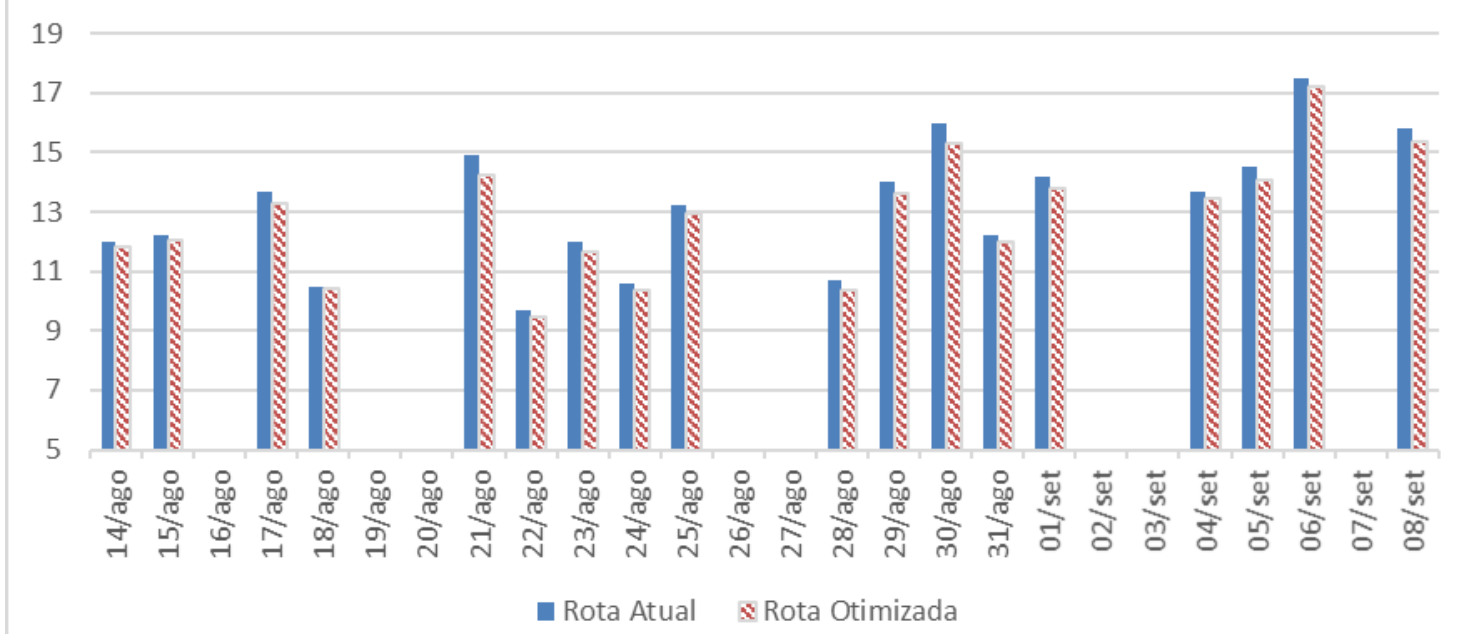

Fonte: Elaborado pelos autores

Como observado no Gráfico 2, nota-se que a rota otimizada possui valores ligeiramente menores que a rota atual. Por isso, para melhor efeito comparativo 
contabilizou-se todo o tempo de atendimento durante o período, a diferença total entre os dois cenários está representada na Tabela 8.

Tabela 8 - Indicador: tempo entre pedido e recebimento

\begin{tabular}{lccc}
\hline \multicolumn{1}{c}{ Indicador } & Rota Atual & $\begin{array}{c}\text { Rota } \\
\text { Otimizada }\end{array}$ & $\begin{array}{c}\text { Redução } \\
\text { (Total) }\end{array}$ \\
\hline $\begin{array}{l}\text { Tempo entre o pedido e o } \\
\text { recebimento }\end{array}$ & $237,4 \mathrm{~h}$ & $231,3 \mathrm{~h}$ & $6,1 \mathrm{~h}$ \\
\hline
\end{tabular}

Fonte: Elaborado pelos autores

A partir da Tabela 8, observa-se que a adoção da roteirização resultaria em uma redução um pouco mais de seis horas no tempo de atendimento, ou seja, mais de um turno de funcionamento da organização, ou ainda, redução em cerca de vinte minutos por rota no processo de atendimento. Dessa forma, entende-se que a diminuição no tempo de atendimento é bastante proveitosa, uma vez que aperfeiçoaria o processo de atendimento aos clientes, melhorando a eficiência e, consequentemente, o nível de serviço da organização. Ademais, esse ganho ou redução está atrelado aos 18 dias de observação que dá uma média de pouco mais de 3 horas por dia. Fazendo uma estimativa anual, seria só multiplicar esse último valor pela quantidade de dias trabalhados por ano $\left(20^{*} 12\right)$, esse valor se aproxima de 720 horas, portanto, estima-se em cerca de 90 dias de ganho.

\subsubsection{Terceiro indicador: custos envolvidos coma distribuição}

Finalmente, para a obtenção do terceiro parâmetro analisado, que é referente aos custos envolvidos com a distribuição dos produtos, calculou-se a porcentagem de redução entre os custos variáveis na rota atual indicada pela Tabela 5 e os custos variáveis da rota otimizada descritos na Tabela 8 . O Gráfico 3, mostra o comparativo entre os dois diferentes cenários: 


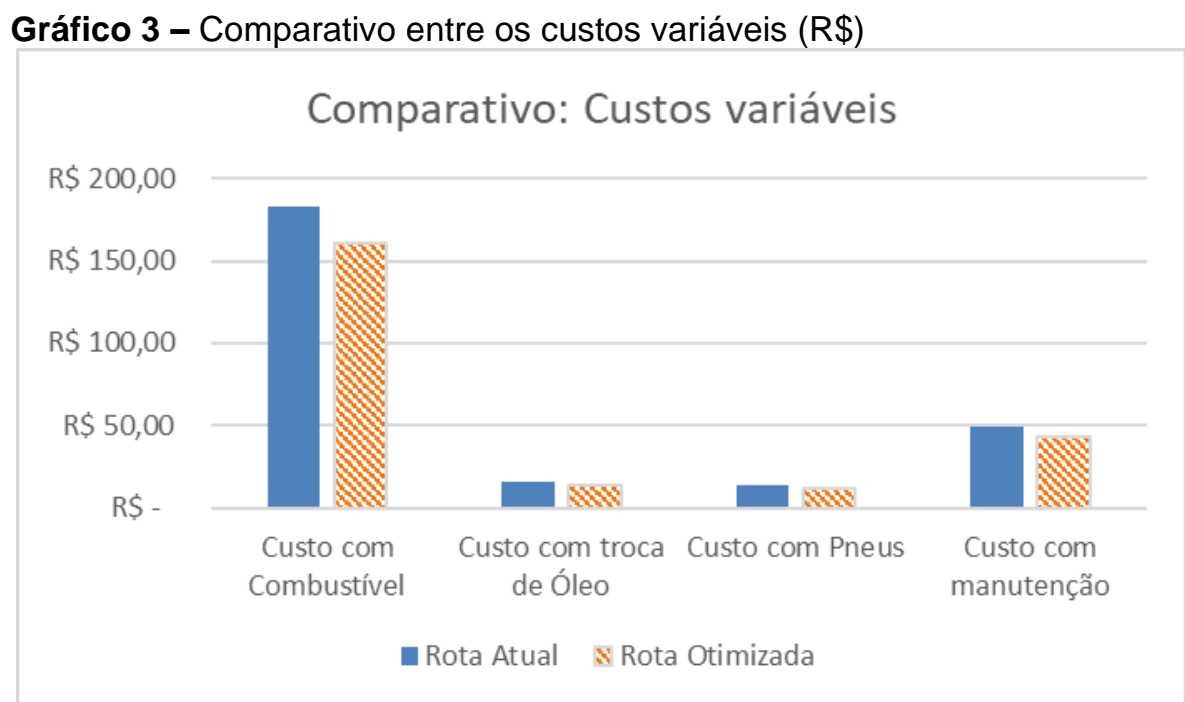

Fonte: Elaborado pelos autores

Conforme exposto no Gráfico 3, verifica-se que a rota otimizada possui valores ligeiramente menores, sendo mais evidente no custo com combustível, o custo de maior parcela de contribuição nos custos variáveis. Nesse sentido, objetivando evidenciar o valor total referente aos custos variáveis somaram-se os valores nos dois cenários, o resultado pode ser verificado na Tabela 9.

Tabela 9 - Indicador: custo envolvido com a distribuição dos produtos

\begin{tabular}{lccc}
\multicolumn{1}{c}{ Indicador } & $\begin{array}{c}\text { Rota } \\
\text { Atual }\end{array}$ & $\begin{array}{c}\text { Rota } \\
\text { Otimizada }\end{array}$ & Redução \\
\hline $\begin{array}{l}\text { Custos envolvidos com a distribuição dos } \\
\text { produtos }(\mathrm{R} \$)\end{array}$ & $\mathrm{R} \$ 262,26$ & $\mathrm{R} \$ 229,90$ & $12,34 \%$ \\
\hline Fonte: Elaborado pelos autores & & &
\end{tabular}

Analisando os resultados contabilizados da Tabela 9 percebe-se que realizando a rota indicada pelo software a distribuidora obteria uma economia monetária de aproximadamente $12,34 \%$ em seus custos variáveis, resultado bastante vantajoso devido a importância que a atividade de distribuição possui para empresas desse segmento.

De igual modo que no tempo de atendimento, si estimar esse ganho em um horizonte de um ano, dessa forma seria a diferença do valor da rota atual com a otimizada divido por 18 dias (1,80 Reais) e si multiplicar esse valor pela quantidade de dias de trabalho em um ano (20*12), daria uma estimativa de aproximadamente de 640 reais por ano de economia. 
Portanto, conclui-se que os dados dos três indicadores evidenciam os impactos positivos da otimização por meio de um software da rota de veículos da empresa em estudo.

\section{CONCLUSÃO}

O presente trabalho situa-se no âmbito da logística de distribuição, tema frequentemente discutido e desafiador para as organizações inseridas em um ambiente globalizado, cada vez mais dinâmico e competitivo. Esse cenário tem exigido que as empresas busquem melhorar seu nível de serviços e ainda, a redução dos custos logísticos. Considerando os fatores mencionados, o estudo analisou o impacto e/ou os benefícios gerados na aplicação da roteirização da frota de veículos em uma empresa distribuidora de produtos de limpeza. Para isso, evidenciou o comparativo entre a rota realizada e rota otimizada pelo software LOGWARE.

Com os resultados do estudo realizado, pode-se constatar que a adoção da roteirização mediante o software logístico apresentou melhorias na logística de transporte, conforme explicitado nos indicadores de distância percorrida pelas rotas (redução de 183,5 km), tempo de atendimento (redução de 6,1 h) e custos variáveis (redução de 12,34\%) durante o período de 18 dias de analise. Isso ocorre, pois, atualmente durante o processo de distribuição de produtos, os locais são percorridos apenas conforme a experiência e o conhecimento dos motoristas de maneira intuitiva, esse fator possibilita maiores deslocamentos para a maioria das rotas traçadas, além de frequentes viagens desnecessárias.

Diante dos resultados, é possível traçar algumas diretrizes tanto para a empresa escolhida quanto aquelas que não fazem uso de softwares para definir suas rotas. Uma proposta, que pode ser entendida como duas ao mesmo tempo, é que as organizações visem investir em profissionais de logística e de software de roteirização, visto que em um período curto houve uma significante redução dos indicares analisados. Outra sugestão está no fato de estender esse estudo por um período mais amplo visando alcançar resultados mais expressivos. 


\section{REFERÊNCIAS}

AMARAL, D. B. M.; SOUZA, A. W.; KENJI OI, Ricardo; CARNEIRO, J. B.; SANTOS, R. M.. Estudo de Roteirização de Veículos com Aplicação da Técnica de Varredura para Cargas Fracionadas. In: SIMPÓSIO DE EXCELÊNCIA EM GESTÃO E TECNOLOGIA, 9., 2014, Rio de Janeiro. [Anais...] 2014.

BALLOU, R. H. Gerenciamento da cadeia de suprimentos: logística empresarial. 5. ed. Porto Alegre: Bookman, 2006.

BALLOU, R. H. Logware: programas de computador selecionados para planejamento logístico. 1999.

BOWESOX, Donald J; CLOSS, D. J; COOPER, M. B.; BOWESOX, J. C.. Gestão Logística da cadeia de suprimentos. 4. ed. Porto Alegres: AMGH, 2014.

CARLOS, F; GORDON, F. Configuration of physical distribution networks. Institute of studies, Universidade da Califórnia. Berkeley. Networks, v.16, p.113-132, 1986. Disponível em: http://onlinelibrary.wiley.com/doi/10.1002/net.3230160202/pdf .Acesso em: 02 jun. 2018. https://doi.org/10.1002/net.3230160202

CARVALHHO, M. Roberto. Gestão dos canais de distribuição. 1. ed. Curitiba: IESDE Brasil S. A, 2009.

CHOPRA, S.; MEINDL, P. Gestão da cadeia de suprimentos: estratégia, planejamento e operações. 4 e.d. São Paulo: Pearson, 2011.

CUNHA, C. B. Aspectos práticos da aplicação de modelos de roteirização de veículos a problemas reais. Revista Transportes, v.8, n.8, p.51-74, 2000.

https://doi.org/10.14295/transportes.v8i2.188

DAVIDSSON, P; HENESEY, L; RAMSTEDT, L; TORNQUIST, J; WERNSTEDT, F. Ananalysis of agent-based approaches to transport logistics. Transportation Research Part C: Emerging Technologies, v.13, n. 4, p. 255-271, 2005.

https://doi.org/10.1016/j.trc.2005.07.002

DORNIER, Philippe Pierreetal. Logística e operações globais: textos e casos. São Paulo: Atlas, 2011.

ENOMOTO, L. M. Análise da distribuição física e roteirização em um atacadista do sul de minas gerais. 2005. 142 p. Dissertação (Mestrado em Ciências em Engenharia de Produção) - Engenharia de produção, Universidade Federal de Itajubá, UNIFEI, Itajubá, 2005. Disponível em: http://saturno.unifei.edu.br/bim/0029652.pdf. Acesso em: 04 abr. 2018. https://doi.org/10.1590/S0103-65132007000100007

ENOMOTO, Leandro Minoru; LIMA, Renato da Silva. Análise da distribuição física e roteirização em uma atacadista. Produção, v.17, n.1, p.094-108, 2007.

GOMES, D. M.; LIMA, D. S.; GARCIA, F. C.; SILVA, H. P.; NASCIMENTO M. R. L. Análise do processo de roteirização em uma empresa atacadista. 2012. Disponível em: http://www.gpslog.com.br/portal/wp-content/uploads/TCCDISTRIBUI\%C3\%87\%C3\%830.pdf. Acesso em: 14 mar. 2018. 
ILOS. Instituto de logística e supply chain. Disponível em:

http://www.ilos.com.br/web/solucoes-por-tema/solucoes-por-tema-custos-logisticos/. Acesso em: 18 abr. 2018.

JACOBS, F. R.; CHASE, R. B. Administração de operações e da cadeia de

suprimentos.13. ed. Porto Alegre: AMGH, 2012.

KAZIMÍROVÁ, I; BUSA, M; PUSKÁS, E. Structure of corporate logistic costs. The International Journal of transport \& logistics. v.15, p.15, 2015.

MARTINS, R. S; VICENTE, J. Gestão logística do transporte de cargas. 1.ed. São Paulo, Atlas, 2015.

MELO, A. C. S.; FERREIRA FILHO, V. J. M.. Sistemas de roteirização e programação de veículos. Pesquisa Operacional, v. 21, n. 2, p. 223 - 232, 2001.

https://doi.org/10.1590/S0101-74382001000200007

MENCHIK, Carlos Roberto. Gestão estratégica de transportes e distribuição. Curitiba: IESDE Brasil S. A, 2010.

NETO, ALBERTO; LIMA, RENATO. Roteirização de veículos de uma rede atacadista com o auxílio de sistemas de informações geográficas (sig). Revista Pesquisa e

Desenvolvimento Engenharia de Produção. n.5, p. 18 - 39, 2006

NOVAES, Antônio Galvão. Logística e gerenciamento da cadeia de distribuição. Rio de Janeiro: Elsevier, 2015.

PEREIRA, L. S. S.; DUARTE, R. B.; FIRMINO, C. R. S.; IRINEU, A. B. M.; COSTA, J. R. A. A.. Otimização de rota na distribuição de produtos em uma empresa de produtos de beleza. In: ENEGEP, 35., 2015. [Anais eletrônicos... ]. Fortaleza, 2015.

REBEZOVA, M; SULIMA, N; SURINOV, R. Development trends of air passenger transport services and service distribution channels. Transport and Telecommunication, v.13, n.2, p.159 - 166, 2012. https://doi.org/10.2478/v10244-012-0013-9

ROSA, Adriano C.. Gestão do transporte na logística de distribuição física: uma análise da minimização do custo operacional. 2007. Dissertação (Mestrado em Curso de Gestão em Desenvolvimento Regional) - Departamento de Economia, Contabilidade e Administração, Universidade Taubaté, UT, São Paulo. Disponível em:

http://www.ppga.com.br/mestrado/2007/rosa-adriano carlos.pdf. Acesso em: 18 abr. 2018.

ROSA, Rodrigo de Alvarenga. Gestão de operações e logística I. Florianópolis:

Departamento de Ciências da Administração - UFSC; [Brasília]: CAPES: UAB, 2011.

RUSHTON, A; CROUCHER, P; BAKER, P. The Hand book of Logistics and Distribution Management: Understanding the Supply Chain. 5. Ed. The charter institute of logistics and transport UK, United Kingdon, 2014.

SANTOS, F. V. A utilização da pesquisa operacional como ferramenta para redução de custos na logística de distribuição: problema de roteamento de veículos capacitados (prvc). 2014. 127 p. Dissertação (Trabalho de conclusão de curso em Engenharia de Produção), Engenharia de Produção, Universidade de Formiga, UNIFOR-MG, Formiga. Disponível em: http://bibliotecadigital.uniformg.edu.br:21015/ispui/handle/123456789/279. Acesso em: 03 abr. 2018. 
SKWARA, Frederico N. Análise da atratividade da indústria de provedores de Serviços logísticos para investimentos de private equity. 2013. 120p. Dissertação (Trabalho de conclusão de curso em Engenharia de Produção), Engenharia de Produção, Escola Politécnica da Universidade de São Paulo, EPUSP-SP, São Paulo. Disponível em: http://pro.poli.usp.br/wp-content/uploads/2013/12/TF vFinal6.pdf. Acesso em: 09 maio 2018.

YASAR, Mehmet. Analysis of the competition between transportation modes from the perspective of competitive dynamics: a study on ankara-Istanbul transportation line.Transport \& Logístics: the International Journal, v.17, n.42, 2017.

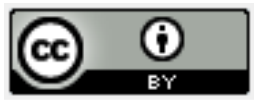

Artigo recebido em: 24/08/2018 e aceito para publicação em: 07/06/2020

DOI:http://dx.doi.org/10.14488/1676-1901.v20i2.3356 